\title{
Hypnotic Suggestions Can Induce Rapid Change in Implicit Attitudes
}

\author{
Pieter Van Dessel and Jan De Houwer \\ Ghent University, Belgium
}

Word count: 2.000

\begin{abstract}
Author Note
PVD and JDH, Department of Experimental Clinical and Health Psychology, Ghent University. PVD is supported by a Postdoctoral fellowship of the Scientific Research Foundation, Flanders (FWO-Vlaanderen). JDH is supported by Methusalem Grant BOF16/MET_V/002 of Ghent University. Correspondence concerning this article should be sent to Pieter.vanDessel@UGent.be
\end{abstract}

This paper is not the copy of record and may not exactly replicate the final, authoritative version of the article as published in Psychological Science. Please do not copy or cite without authors permission. The final article will be available, upon publication, via its DOI. 


\begin{abstract}
We sometimes evaluate our environment (e.g., persons, objects, or situations) in an automatic fashion. These automatic or implicit evaluations are often considered to be based on qualitatively distinct mental processes compared to more controlled or explicit evaluations. Important evidence for this claim comes from studies showing that implicit evaluations do not change as the result of counter-attitudinal information, in contrast to their explicit counterparts. We examined the impact of counter-attitudinal information on implicit evaluations in two experiments $(N=60$, $N=72$ ) that included an innovative manipulation: hypnotic suggestions of enhanced processing of upcoming counter-attitudinal information. Both experiments indicated that hypnotic suggestions facilitated effects of counter-attitudinal information on implicit evaluations. These findings extend recent evidence for rapid revision of implicit evaluations on the basis of counterattitudinal information and support the controversial idea that belief-based processes underlie not only explicit but also implicit evaluations.
\end{abstract}

Keywords: implicit attitudes, automatic evaluation, implicit-explicit dissociation, counterattitudinal information, hypnotic suggestions 


\section{Hypnotic Suggestions Can Induce Rapid Change in Implicit Attitudes}

When we encounter a specific object (e.g., a pizza) or person (e.g., Donald Trump), this can trigger an evaluative response (e.g., a smile, a frown) in a spontaneous or automatic manner. Research suggests that these automatic or implicit evaluations are important determinants of behavior (see Cameron, Brown-Iannuzzi, \& Payne, 2012; Friese, Hofmann, \& Schmidt, 2008, for reviews). For instance, implicit evaluations of unhealthy foods can bias food choices (Marty et al., 2017) and implicit evaluations of politicians can affect voting behavior (Raccuia, 2016).

Interestingly, implicit evaluations do not always accord with evaluations that arise in a more controlled manner (explicit evaluations; e.g., self-reported ratings of liking) (see Petty \& Briñol, 2009, for a review). Most prominently, information that contradicts prior evaluations (i.e., counter-attitudinal information) sometimes leads to rapid changes in explicit but not implicit stimulus evaluations. In one of the most cited studies in the field of attitude research in the last twenty years, Gregg, Seibt, and Banaji (2006) installed evaluations of two fictitious social groups (Niffites and Luupites) by informing participants that one group had positive traits and the other group had negative traits. When participants afterwards saw information that ascribed traits of the opposite valence to Niffites and Luupites, implicit evaluations of the groups did not change even though explicit evaluations completely reversed.

This intriguing finding of a dissociative effect of counter-attitudinal information on implicit and explicit evaluations is considered one of the most important pieces of evidence for the idea that two qualitatively distinct processes underlie implicit and explicit evaluation. Dualprocess theories of evaluation typically postulate that implicit evaluation results from the automatic activation of learned associations in memory, whereas explicit evaluation depends on belief-based processes such as the validation of activated information (e.g., Rydell \& McConnell, 
2006; Strack \& Deutsch, 2004). Associations are often construed as links between mental representations (e.g., a link between representations of pizza and of positive valence) that, once established, cannot be simply erased from memory and might therefore drive implicit evaluation even when the implied evaluation is no longer endorsed explicitly.

The idea that inherently stable mental associations underlie implicit evaluations is often used to explain dissociations between implicit and explicit evaluation (e.g., in person perception: Okten, 2018; racial prejudice: James, 2018; addiction: Wiers et al., 2017). Moreover, it has directed intervention research that aims to modify unwanted implicit preferences. Changing implicit evaluations is often assumed to require repeated pairings of stimuli with valenced events because this produces gradual changes in associations (Rydell \& McConnell, 2006). Intervention studies have therefore mainly used procedures that continuously pair target stimuli with valenced stimuli (evaluative conditioning: EC; Hofmann, De Houwer, Perugini, \& Crombez, 2010) or valenced responses (approach-avoidance training: Van Dessel, Hughes, \& De Houwer, 2018a).

Importantly, however, recent studies have challenged the idea that the modification of implicit evaluations requires repeated pairings by demonstrating that counter-attitudinal information can sometimes induce rapid change in implicit evaluation. For instance, participants who had learned positive information about a person named Bob, exhibited a rapid negative shift in implicit evaluations of Bob when they learned new information that they considered more diagnostic of Bob's true character (e.g., that Bob was a convicted child molester; Cone \& Ferguson, 2015). Other studies indicated that the extent to which counter-attitudinal information is believable and allows reinterpretation of the initial information also moderates rapid change in implicit evaluation (see Cone, Mann, \& Ferguson, 2018, for an overview). These results suggest that belief-based processes contribute to implicit evaluation and have bolstered the innovative 
idea that both implicit and explicit evaluations constitute evaluative responses that are triggered by beliefs about the valence of a stimulus that are readily available under the different measurement conditions (Van Dessel, Hughes, \& De Houwer, 2018a).

To systematically examine the belief-based processes that underlie implicit evaluation, one particularly useful method might be hypnosis. Hypnosis is commonly defined as "an event or ritual between a hypnotist and a hypnotic subject in which both agree to use suggestion to bring about a change in perception or behavior" (Bernheim, 1965). This ritual typically involves (1) an induction during which the hypnotist invites the subject to experience a state in which they focus on, accept, and act in line with verbal suggestions that are provided by the hypnotist and (2) the verbal suggestions (Oakley \& Halligan, 2013). Research has shown that hypnotic suggestions can sometimes facilitate responding in-line with specific suggestions even when they provide information that contrasts with participants' prior beliefs (Raz \& Shapiro, 2002). As such, hypnosis has often been used with the aim of changing a person's current beliefs in order to promote new, adaptive behavior (e.g., the inhibition of pain responses in pain management: Patterson \& Jensen, 2003). Previous studies have found that hypnotic suggestions can influence even highly automatic behavior (see Lifshitz, Bonn, Kashem, \& Raz, 2013), yet, no study to date has examined effects on implicit evaluation. Importantly, however, if beliefs (rather than learned associations) determine implicit evaluation, then providing hypnotic suggestions could be a very potent method for changing even highly robust implicit evaluations because it is a very potent method for changing beliefs. Hence, our research can shed new light on the conditions under which implicit evaluations change and thus the nature of the underlying representations.

In the current research, we tested effects of hypnotic suggestions on implicit evaluation in two paradigms that are known to generate robust changes in explicit but not implicit evaluations 
as the result of counter-attitudinal information. Experiment 1 adopted the procedure of the influential Gregg et al. (2006) studies, testing effects of counter-attitudinal information on evaluations of fictitious social groups that were installed via a historical narrative. Experiment 2 probed effects of counter-attitudinal information on evaluations of two unfamiliar persons as installed via EC, which capitalizes on repeated pairings and is therefore considered the most direct way to install strong implicit evaluations from dual-process perspectives (Rydell \& McConnell, 2006; Hu, Gawronski, \& Balas, 2017). In both experiments, we examined the relative resistance of implicit and explicit evaluations to verbal counter-attitudinal information. Importantly, we provided half the participants with hypnotic suggestions that they would strongly process and incorporate the counter-attitudinal information. We predicted that these hypnotic suggestions of enhanced processing of counter-attitudinal information would facilitate effects of this information not only on explicit but also on implicit evaluations.

\section{Method}

\section{Participants}

A total of 60 and 72 native Dutch-speaking undergraduates were recruited at Ghent University, Belgium, for Experiments 1 and 2, respectively. Initial sample size was determined based on an a priori power analysis such that we would have sufficient power (i.e., power $>0.80$ ) to detect an effect of similar magnitude to prior results of Gregg et al. (2006), for Experiment 1, and to detect an effect of similar magnitude to the effect observed in Experiment 1, for Experiment $2 .{ }^{1} \mathrm{We}$ ensured sufficient statistical power to also detect smaller effects by planning sample size increases until decisive evidence was obtained (as indicated by the Bayes Factor) for the presence

\footnotetext{
${ }^{1}$ The Editor brought to our attention that there are reasons to believe that published effects often exaggerate the size of true effects due to publication bias and other factors (see e.g., Szucs \& Ioannidis, 2017).
} 
or absence of an effect (Schönbrodt, Wagenmakers, Zehetleitner, \& Perugini, 2017) but increases proved unnecessary. Prior to data-collection, target sample size was pre-registered together with the study design, data-analytic plans, and experimental hypotheses. The pre-registered plans, raw data, experimental and analytic scripts are available at https://osf.io/wkjp6/.

Because suggestibility can be a potent moderator of hypnosis effects (see Raz et al., 2006), all participants in the Ghent University participation pool completed the Multidimensional Iowa Suggestibility Scale Brief (MISS; Kotov, Bellmann, \& Watson, 2004) and only participants with the $50 \%$ highest scores were invited for participation in Experiment 1. To facilitate generalizability, Experiment 2 measured suggestibility but did not pre-select participants on suggestibility scores.

\section{Procedure}

Upon entering the research lab, participants received information about the phenomenology of hypnosis (standard hypnosis rationale: Shor \& Orne, 1962). Next, participants provided informed consent and were seated in front of a computer screen.

Evaluation induction. In Experiment 1, participants were informed that they would learn about a real historical conflict between two social groups (i.e., Niffites and Luupites) of which the true identities were concealed. They then read a story describing one group as civilized and constructive (positive induction group) and the other group as aggressive and destructive (negative induction group) (Gregg et al., 2006; Experiment 4).

In Experiment 2, participants were informed that they would learn about two persons (i.e., Bob and Jan) whose pictures were presented below the instructions (images of neutral valence taken from the Chicago Face Database). Participants were then subjected to an EC procedure (adapted from $\mathrm{Hu}$ et al., 2017). They were informed that they would see pictures and words 
presented on the screen and that they should be attentive to these presentations because they would be asked questions about them afterwards. There were $80 \mathrm{EC}$ trials and each trial involved the presentation of one target person together with a positive picture or word (positive induction person) or the other person together with a negative picture or word (negative induction person). Presentations were displayed for $1000 \mathrm{~ms}$, the inter-trial interval was $2000 \mathrm{~ms}$. In contrast to Experiment 1, we collected evaluations at two separate times in Experiment 2. After the EC procedure, participants completed implicit and explicit evaluation measures of the two target persons for a first time (measurement details are described below).

Hypnosis induction. After the evaluation induction, participants were led to another room where they met a trained hypnotist (the first author), who asked them to sit down in a comfortable chair. Half of the participants (hypnosis condition) then received standard hypnotic induction via a verbal suggestion induction procedure (Shor \& Orne,1962). This procedure consisted of elaborate instructions to focus on the words provided by the hypnotist, experience a state of deep relaxation, and let happen what the hypnotist told them would happen. The induction procedure ended with a short test of hypnotic suggestibility in which participants were asked to imagine arm lowering and arm immobilization and the hypnotist scored how well they complied with these suggestions. After the hypnosis induction procedure, participants were given the following hypnotic suggestions (translated from Dutch): "You will now receive information that you will process more strongly than you normally can. Please remember well that the information that you will hear next, will sink in more deeply than is typically the case." An English translation of all the instructions that were given to participants in the hypnosis condition can be found in the Supplementary Online Material. 
In accordance with Raz et al. (2006), the other participants (relaxation control condition) were asked to close their eyes, perform several relaxation exercises, and listen carefully to the information they would hear next. This condition matched the hypnosis condition on important factors such as the person providing the information, the place, and the approximate timing of the event, but not the hypnosis, that is, the ritual that involved (1) providing instructions to focus on the hypnotist's words and (2) the suggestion of enhanced processing of the upcoming information.

Counter-attitudinal information. In Experiment 1, half of the participants in both the relaxation and hypnosis conditions were told a narrative describing how, following the events they learned about previously, the positive induction group (e.g., Niffites) became hateful and aggressive (e.g., engaging in terrorist attacks) and the negative induction group (e.g., Luupites) became peaceful and noble (e.g., donating their possessions). The other participants heard a control narrative describing flora and fauna in Niffites and Luupites' residential areas. Texts were adopted from Gregg et al. (2006).

In Experiment 2, all participants were told the following information (translated from Dutch): "I will now tell you about the two people you learned about previously, that is, Bob and Jan. I want you to know that Jan is a very nice and friendly person whereas Bob is very unpleasant and mean."

Implicit and Explicit Evaluation Measurement. After participants were instructed to open their eyes to terminate the relaxation or hypnosis phase, participants went back to the evaluation induction room to complete evaluation measures. The order of implicit and explicit evaluation measurement was counterbalanced across conditions. In Experiment 1, measurement followed Gregg et al. (2006). An Implicit Association Test (IAT; Greenwald, McGhee, \& 
Schwartz, 1998) was used to measure implicit evaluations of Niffites and Luupites. In this task, participants categorized 24 attribute words (e.g., wonderful, painful) as 'positive' or 'negative' and eight names of Niffites and Luupites as their respective group labels. In one experimental block, stimuli related to one group (e.g., Niffites) and positive shared a response key and stimuli related to the other group (e.g., Luupites) and negative shared a second response key. In the other experimental block, response key assignment was reversed. Both experimental blocks consisted of 48 trials. It is assumed that faster responding in one block (e.g., the Niffites-positive and Luupites-negative block) is indicative of a more positive automatic (e.g., fast, unintentional) evaluation in line with the category pairings (e.g., Niffites are evaluated more positively than Luupites). Explicit evaluations of Niffites and Luupites were measured by asking participants to rate both groups using four 7-point semantic differentials with the following endpoints: horriblewonderful, unpleasant-pleasant, bad-good, and corrupt-virtuous.

In Experiment 2, evaluations of Bob and Jan were measured in accordance with Peters and Gawronski (2011). Implicit evaluations were probed with an Affect Misattribution Procedure (AMP; Payne, Cheng, Govorun, \& Stewart, 2005) consisting of 60 trials. Each trial, participants were presented with a prime stimulus that depicted the face of Bob or Jan (75ms), followed by a blank screen (125ms), a Chinese ideograph (100ms), and a black-and-white pattern mask. Participants were asked to indicate if they considered the Chinese ideograph more or less visually pleasant than average by pressing either "E" or "I", respectively. A higher proportion of positive evaluations of Chinese ideographs with Jan than with Bob as prime stimulus is assumed to reflect a more positive evaluation of Jan. This evaluation is often considered automatic (in the sense of unintentional) because participants are instructed to ignore the pictures of Bob and Jan. Explicit evaluations of Bob and Jan were measured with three self-report items probing likeability, 
friendliness, and trustworthiness (randomized order). Responses were provided on 7-point rating scales ranging from 1 (not at all) to 7 (very much).

Final Questions. Participants indicated (a) whether their evaluative ratings reflected actual liking or demand compliance, (b) the extent to which they had felt under hypnosis (hypnosis condition), (c) their perceived believability of the historical texts (Experiment 1), and (d) observed contingencies in the EC task (Experiment 2). Participants in Experiment 2 also completed a measure of suggestibility (MISS) and psychological reactance (Hong \& Faedda, 1996). Finally, participants also indicated the implied valence of the counter-attitudinal information. All participants (except for two hypnosis condition participants in Experiment 2) answered these questions correctly, indicating that participants in both conditions had been attentive to the information. Exclusion of participants who gave an incorrect response did not change the significance level of any of the reported findings.

\section{Results}

\section{Experiment 1}

Implicit evaluation. In accordance with Gregg et al. (2006), trials with latencies above 3000ms or below 300ms $(1.2 \%)$ were excluded and trial latencies were transformed by dividing 1000 by the respective latencies. IAT scores were computed by subtracting participants' mean transformed latencies in the compatible IAT block (positive induction group and positive words assigned to the same key) from mean transformed latencies in the incompatible IAT block (positive induction group and negative words assigned to the same key). Overall, IAT scores were higher than zero, indicating an implicit preference for the positive induction group over the negative induction group $(M=0.14, S D=0.20), t(59)=5.32, p<.001,95 \% \mathrm{CI}=[0.09,0.19], d=$ $0.69,95 \% \mathrm{CI}$ of $d=[0.40,0.97], B F_{1}=9782$. An analysis of variance (ANOVA) on IAT scores 
revealed a main effect of Counter-Attitudinal Information, $F(1,56)=9.20, p=.004, \chi^{2}=0.16$, $B F_{1}=3.29$, and the predicted interaction with Hypnosis, $F(1,56)=5.39, p=.024, \chi^{2}=0.09, B F_{1}$ $=2.60$. Planned contrasts revealed that participants in the hypnosis condition exhibited lower IAT scores when they had learned counter-attitudinal information $(M=0.01, S D=0.20)$ than when they had learned control information $(M=0.22, S D=0.19), t(28)=-2.92$, one-tailed $p=.003$, $95 \% \mathrm{CI}=[\operatorname{Inf},-0.09], d=1.07,95 \% \mathrm{CI}$ of $d=[0.21,1.07], B F_{l}=13.44$. In contrast, and in-line with Gregg et al. (2006), IAT scores of participants in the relaxation condition were not moderated by the type of information (counter-attitudinal information: $M=0.17, S D=0.22$; control information: $M=0.16, S D=0.13), t(28)=0.26$, one-tailed $p=.60,95 \% \mathrm{CI}=[-\mathrm{Inf}, 0.13]$, $d=0.10,95 \%$ CI of $d=[-0.65,0.84], B F_{0}=3.45$. Analyses on IAT scores computed with the D scoring algorithm that incorporates errors in IAT scores produced similar results (see Supplementary Online Material). For the sake of conciseness, pre-registered analyses that are not of focal interest are also reported in the Supplementary Online Material.

Explicit evaluation. Explicit ratings were collapsed into one score for each group $($ Cronbach's Alpha $=.96)$. Explicit evaluation scores were computed by subtracting rating scores for the negative induction group from scores for the positive induction group. Overall, explicit evaluation scores indicated a preference for the positive induction group $(M=1.21, S D=4.53)$, $t(59)=2.06, p=.043,95 \% \mathrm{CI}=[0.04,2.38], d=0.27,95 \% \mathrm{CI}$ of $d=[0.01,0.52], B F_{1}=1.02$. The ANOVA revealed a main effect of Counter-Attitudinal Information, $F(1,56)=123.11, p<$ $.001, \chi^{2}=0.51, B F_{1}>1000$, and an (unexpected) interaction with Hypnosis, $F(1,56)=5.85, p=$ $.019, \chi^{2}=0.02, B F_{1}=1.03$. Participants in the hypnosis condition preferred the positive induction group less when counter-attitudinal information was presented $(M=-3.85, S D=2.65)$ than when control information was presented $(M=5.23, S D=2.09), t(28)=-10.42$, one-tailed $p<.001$, 
$95 \% \mathrm{CI}=[-\operatorname{Inf},-7.60], d=3.80,95 \% \mathrm{CI}$ of $d=[2.17,5.41], B F_{1}>1000$. Similarly, and in-line with Gregg et al. (2006), scores of participants in the relaxation condition were also moderated by counter-attitudinal information (counter-attitudinal information: $M=-1.42, S D=1.89$; control information: $M=4.87, S D=2.26), t(28)=-8.25$, one-tailed $p<.001,95 \% \mathrm{CI}=[-\mathrm{Inf},-4.99]$. Notably, this effect was reduced compared to the hypnosis condition, $d=3.01,95 \%$ CI of $d=$ $[1.63,4.36], B F_{1}>1000$. Results of exploratory (correlational) analyses involving IAT scores, rating scores, self-reported hypnosis scores, and hypnotic suggestibility scores are described in the Supplementary Online Material.

\section{Experiment 2}

Implicit evaluation. Scores for the pre- and post-manipulation AMP were computed by subtracting the percentage of 'pleasant' responses on trials with the negative induction person from the percentage of 'pleasant' responses on trials with the positive induction person. An ANOVA on AMP scores revealed a main effect of Time, $F(1,68)=35.07, p<.001, \chi^{2}=0.34$, $B F_{1}>1000$, and a marginally significant interaction effect of Time and Hypnosis, $F(1,68)=3.52$, $p=.065, \chi^{2}=0.05, B F_{1}=1.09$. Planned contrasts did not reveal a significant difference between the hypnosis $(M=0.25, S D=0.28)$ and relaxation condition $(M=0.25, S D=0.38)$ at Time 1 , $t(70)=-0.01$, one-tailed $p=.50,95 \% \mathrm{CI}=[-\operatorname{Inf}, 0.13], d=0.00,95 \% \mathrm{CI}$ of $d=[-0.23,0.23], B F_{0}$ $=4.50$. In contrast, and most crucially, at Time 2, AMP scores were lower in the hypnosis condition $(M=-0.24, S D=0.43)$ than in the relaxation condition $(M=-0.01, S D=0.40), t(70)=$ -2.42 , one-tailed $p=.009,95 \% \mathrm{CI}=[-\operatorname{Inf},-0.07], d=0.41,95 \% \mathrm{CI}$ of $d=[0.17,0.65], B F_{1}=$ 4.57. Interestingly, AMP scores were reduced from Time 1 to Time 2 in both hypnosis and relaxation groups, $t \mathrm{~s}<-3.01, p \mathrm{~s}<.005, B F_{l} \mathrm{~s}>17.08$. 
Explicit evaluation. Explicit ratings were collapsed into one score for Bob and Jan at each time of assessment (Cronbach's Alpha = .94). Explicit evaluation scores were computed by subtracting rating scores for the negative induction person from scores for the positive induction person. The ANOVA on explicit evaluation scores revealed a main effect of Time, $F(1,68)=$ 70.03, $p<.001, \chi^{2}=0.51, B F_{1}>1000$, but no interaction of Time $\mathrm{x}$ Hypnosis, $F(1,68)=2.67, p$ $=.11, \chi^{2}=0.04, B F_{0}=1.31$. At Time 1 , we did not observe a difference between the hypnosis $(M$ $=1.99, S D=1.72)$ and relaxation condition $(M=1.82, S D=1.83), t(70)=0.40$, one-tailed $p=$ $.65,95 \% \mathrm{CI}=[-\mathrm{Inf}, 0.87], d=0.09,95 \% \mathrm{CI}$ of $d=[-0.14,0.32], B F_{0}=6.49$. At Time 2, participants had lower scores in the hypnosis condition $(M=-1.38, S D=2.44)$ than in the relaxation condition $(M=-0.44, S D=2.11), t(70)=1.74$, one-tailed $p=.043,95 \% \mathrm{CI}=[-\mathrm{Inf}$, $0.04], d=0.34,95 \%$ CI of $d=[0.10,0.58], B F_{1}=2.51$.

\section{Discussion}

People sometimes evaluate stimuli in an automatic manner and changing these implicit evaluations has often proven difficult (e.g., Gregg et al., 2006; Lai et al., 2014). For a long time, the dominant explanation was that implicit evaluations reflect the automatic activation of highly stable mental associations that are insensitive to rational reasoning and that changing implicit evaluations therefore requires repeated pairings of stimuli and valenced events (e.g., Rydell \& McConnell, 2006). Current results contrast with this view. First, we observed rapid change in implicit evaluations as the result of a single piece of counter-attitudinal information. Second, we extend previous reports of similar effects (see Cone et al., 2018) by showing that hypnotic suggestions of enhanced processing of the counter-attitudinal information moderated this effect. These findings have important implications. 
On a theoretical level, the current results support the idea that belief-based processes determine implicit evaluation (De Houwer, 2014; Van Dessel, Hughes, \& De Houwer, 2018a). Previous studies found that implicit evaluations can be readily updated on the basis of counterattitudinal information that is much more diagnostic than the initial information (e.g., 'Bob is a child molester': Cone et al., 2015). We observed rapid change in implicit evaluation without requiring this type of information, by providing hypnotic suggestions about the impact of upcoming counter-attitudinal information. Because these suggestions did not include any stimulus information, this effect cannot be explained with changes in mental associations if it is assumed that these changes require pairings of the target stimuli with valenced information (Rydell \& McConnell, 2006; but see Gawronski \& Bodenhausen, 2006, for an alternative view). Instead, the observed change in implicit evaluations might reflect the impact of newly learned beliefs on implicit evaluation. We recently proposed that implicit evaluations result from automatic inferences and low-level action predictions (active inference) that take into account readily available information (Van Dessel, Hughes, \& De Houwer, 2018a). For instance, the presentation of Niffites names in an implicit evaluation task leads to the prediction and resulting execution of a 'positive' response when participants can easily retrieve information about positive characteristics of Niffites. Hypnotic suggestions about the enhanced impact of counterattitudinal information might lead to the formation of an easily accessible belief that strongly biases implicit evaluations.

In this inferential framework, dissociations between implicit and explicit evaluations are thought to arise as the result of processes operating during retrieval (i.e., at the time of evaluation) rather than during learning. Because implicit evaluation measures typically provide less opportunity and motivation to engage in a comprehensive validation of activated information 
(Gawronski \& Bodenhausen, 2006), implicit evaluations might be influenced more strongly by information that is easy to retrieve (Van Dessel, Hughes, \& De Houwer, 2018a). Experiment 1 replicated the finding of Gregg et al. (2006) that counter-attitudinal information impacts differently on implicit (no effect) and explicit evaluation (reversal). One possible explanation is that the counter-attitudinal information facilitated automatic retrieval of the evaluation supported by the initial stimulus information because it referred to this information. As a result, the initial information more strongly determined implicit evaluation. In contrast, the instruction to provide a thoughtful opinion during explicit evaluation facilitated expression of the more recent counterattitudinal information in explicit evaluation, leading to the observed dissociation. Note that dualprocess theories which assume that belief-based processes moderate association formation can also explain such dissociations (Gawronski \& Bodenhausen, 2006).

On a practical level, our results support and extend recent evidence that robust implicit evaluations can be changed quickly on the basis of evaluative learning procedures that are designed to maximize belief-based learning (e.g., the presentation of believable and diagnostic verbal information: Cone \& Ferguson, 2015; Van Dessel, Ye, \& De Houwer, 2018). This is crucial information for intervention research aimed at changing automatic evaluations that might have unwanted effects on behavior (e.g., in the context of addiction: Wiers et al., 2017, phobia: Jones et al., 2013; depression: Becker et al., 2016). Most importantly, it supports a shift in focus from procedures that draw on repeated pairings to procedures that facilitate durable changes in beliefs. A recent study illustrates the applied potential of this novel approach, revealing stronger effects of a belief-based compared to a pairing-based training procedure for changing implicit evaluations (and consumption) of unhealthy foods (Van Dessel, Hughes, \& De Houwer, 2018b). 
The current results also established a novel procedure for changing implicit evaluations: via hypnotic suggestions that focus on the impact of upcoming information. They extend evidence that hypnotic suggestion can induce changes in automatic responses into the attitudes domain (Lifshitz et al., 2013) and support the idea that hypnosis might enable the formation of new beliefs that are (automatically) integrated in action (e.g., on the basis of low-level action predictions: Jamieson, 2016). Our results can also inform clinical practice, where hypnotic suggestions (which usually do not focus on the impact of upcoming information) have already been used for the treatment of unwanted stimulus-related behavior such as anxious and addictive behavior (with unclear effectiveness: see Pelissolo, 2016, Barnes et al., 2010, for relevant reviews).

\section{Constraint on generality}

Results were obtained in a sample of undergraduate Ghent university students. The fact that these subjects were receptive to the idea of hypnosis could moderate observed effects of the hypnotic suggestions. Effects might also be contingent on characteristics of the hypnotist that facilitated or impeded agreement between hypnotist and participant and on the specific initial and counter-attitudinal information that was provided. The effect of hypnotic suggestions on implicit evaluations was reduced in Experiment 2, possibly because the initial information provided a weaker basis for robust implicit evaluations in the control group. Finally, implicit evaluations

were inferred on the basis of responses in specific measures (Experiment 1: IAT; Experiment 2: AMP). Although it is reassuring to see similar effects on these two measures that are known to differ in important ways (Gawronski \& De Houwer, 2014), it is possible that effects might not generalize to other implicit evaluation measures. We have no reason to believe that results depend on other characteristics of participants, materials, or context. 
IMPLICIT EVALUATION AND HYPNOTIC SUGGESTIONS 


\section{Author contributions}

Both authors were involved in developing the study concept and contributed to the design. Data collection and data-analyses were performed by P. Van Dessel. P. Van Dessel drafted the manuscript. J. De Houwer provided critical revisions. Both authors approved the final version of the manuscript for submission. 


\section{References}

Barnes, J., Dong, C.Y., McRobbie, H., Walker, N., Methta, M., \& Stead, L.F. (2010). Hypnotherapy for smoking cessation. Cochrane Database Systematic Reviews, 10, CD001008.

Becker, E. S., Ferentzi, H., Ferrari, G., Möbius, M., Brugman, S., Custers, J., et al. (2016). Always approach the bright side of life: A general positivity training reduces stress reactions in vulnerable individuals. Cognitive Therapy and Research, 40, 57-71.

Bernheim, H. (1965). Hypnosis and suggestion in psychotherapy (C.A. Herter, Trans.). New Hyde Park, New York: University Books. (Original work published 1887)

Cameron, C. D., Brown-Iannuzzi, J. L., \& Payne, B. K. (2012). Sequential priming measures of implicit social cognition: A meta-analysis of associations with behavior and explicit attitudes. Personality and Social Psychology Review, 4, 330-350.

Cone, J., \& Ferguson, M. J. (2015). He Did what?: The Role of Diagnosticity in Revising Implicit evaluations. Journal of Personality and Social Psychology, 108, 37-57.

Cone, J., Mann, T. C., \& Ferguson, M. J. (2018). Can we change our implicit minds? New evidence for how, when, and why implicit impressions can be rapidly revised. Advances in Social Psychology.

De Houwer, J. (2014). A Propositional Model of Implicit Evaluation. Social and Personality Psychology Compass, 8, 342-353.

Friese, M., Hofmann, W., \& Schmitt, M. (2008). When and why do implicit measures predict behaviour? Empirical evidence for the moderating role of opportunity, motivation, and process reliance. European Review of Social Psychology, 19, 285-338. 
Gawronski, B., \& Bodenhausen, G. V. (2006). Associative and propositional processes in evaluation: An integrative review of implicit and explicit attitude change. Psychological Bulletin, 132, 692-731.

Gawronski, B., \& De Houwer, J. (2014). Implicit measures in social and personality psychology. In H. T. Reis, \& C. M. Judd (Eds.), Handbook of research methods in social and personality psychology (2nd edition, pp. 283-310). New York: Cambridge University Press.

Greenwald, A. G., McGhee, D. E., \& Schwartz, J. L. (1998). Measuring individual differences in implicit cognition: the implicit association test. Journal of Personality and Social Psychology, 74, 1464-80.

Gregg, A. P., Seibt, B., \& Banaji, M. R. (2006). Easier done than undone: asymmetry in the malleability of implicit preferences. Journal of Personality and Social Psychology, 90, 120.

Hofmann, W., De Houwer, J., Perugini, M., Baeyens, F., \& Crombez, G. (2010). Evaluative conditioning in humans: a meta-analysis. Psychological Bulletin, 136, 390-421.

Hong, S. M., \& Faedda, S. (1996). Refinement of the Hong Psychological Reactance Scale. Educational \& Psychological Measurement, 56, 173-182.

Hu, X., Gawronski, B., \& Balas, R. (2017). Propositional versus dual-process accounts of evaluative conditioning: I. The Effects of Co-Occurrence and Relational Information on Implicit and Explicit Evaluations. Personality and Social Psychology Bulletin, 43, 43, 1732. 
James, L. (2018), The stability of implicit racial bias in police officers, Police Quarterly, 21, 3052.

Jamieson, G. A. (2016). A unified theory of hypnosis and meditation states: The interoceptive predictive coding approach. In A. Raz \& M. Lifshitz (Eds.), Hypnosis and meditation: Towards an integrative science of conscious planes (pp. 313-342). New York, NY, US: Oxford University Press.

Jones, C. R., Vilensky, M. R., Vasey, M. W., \& Fazio, R. H. (2013). Approach behavior can mitigate predominately univalent negative attitudes: Evidence regarding insects and spiders. Emotion, 13, 989-996.

Kotov, R.I., Bellman, S.B., Watson, D.B. (2004) Multidimensional Iowa Suggestibility Scale (MISS) Brief Manual. Stoneybrook Medicine website. Retrieved from http://www.hsc.stonybrook.edu/som/psychiatry/docs/MISSBriefManual.pdf

Lai, C.K., Marini, M., Lehr, S.A., Cerruti, C. et al. (2014). Reducing implicit racial preferences: I. A comparative investigation of 17 interventions. Journal of Experimental Psychology: General, 143, 1765-1785.

Lifshitz, M., Bonn, A. N., Kashem, I.F., \& Raz, A. (2013). Using suggestion to modulate automatic processes: From Stroop to McGurk and beyond. Cortex, 49, 463-473.

Marty, L., Miguet, M., Bournez, M., Nicklaus, S., Chambaron-Ginhac, S., Monnery-Patris, S. (2017). Do hedonic- versus nutrition-based attitudes toward food predict food choices? a cross-sectional study of 6- to 11-year-olds. International Journal of Behavioral Nutrition and Physical Activity, 14, 162-172. 
Oakley, D. A., \& Halligan, P. W. (2013). Hypnotic suggestion: Opportunities for cognitive neuroscience. Nature Reviews Neuroscience, 14, 565-576.

Okten, I.O. (2018). Studying First Impressions: What to Consider? APS Observer, 31.

Patterson, D.R., \& Jensen, M. (2003). Hypnosis and clinical pain. Psychological Bulletin, 29, $495-521$.

Payne, B. K., Cheng, C. M., Govorun, O., \& Stewart, B. D. (2005). An inkblot for attitudes: Affect misattribution as implicit measurement. Journal of Personality and Social Psychology, 89, 277-293.

Pelissolo, A. (2016). Hypnosis for anxiety and phobic disorders: A review of clinical studies. Presse Medicale, 45, 284-290.

Peters, K. R., \& Gawronski, B. (2011). Are we puppets on a string? Comparing the impact of contingency and validity on implicit and explicit evaluations. Personality and Social Psychology Bulletin, 37, 557-569.

Petty, R. E., \& Briñol, P. (2009). Implicit ambivalence: A meta-cognitive approach. In R. E. Petty, R. H. Fazio, \& P. Briñol (Eds.), Attitudes: Insights from the new implicit measures (pp. 119-161). New York: Psychology Press.

Raccuia, L. (2016). Single-Target Implicit Association Tests (ST-IAT) Predict Voting Behavior of Decided and Undecided Voters in Swiss Referendums. PLOS ONE, 11: e0163872.

Raz, A., Kirsch, I., Pollard, J., \& Nitkin-Kaner, Y. (2006). Suggestion reduces the stroop effect. Psychological Science, 17, 91-95

Raz, A., \& Shapiro, T. (2002). Hypnosis and neuroscience: a cross talk between clinical and cognitive research. Archives of General Psychiatry, 59, 85-90. 
Rydell, R. J., \& McConnell, A. R. (2006). Understanding implicit and explicit attitude change: A systems of reasoning analysis. Journal of Personality and Social Psychology, 91, 9951008.

Schönbrodt, F. D., Wagenmakers, E.-J., Zehetleitner, M., \& Perugini, M. (2017). Sequential hypothesis testing with Bayes factors: Efficiently testing mean differences. Psychological Methods, 22, 322-339.

Shor, R. E., \& Orne, E. C. (1962). Harvard group scale of hypnotic susceptibility, Form A. Palo Alto, CA: Consulting Psychologists Press.

Strack, F., \& Deutsch, R. (2004). Reflective and impulsive determinants of social behavior. Personality and Social Psychology Review, 8, 220-247.

Szűcs, D., \& Ioannidis, J. P. A. (2017). Empirical assessment of published effect sizes and power in the recent cognitive neuroscience and psychology literature. PLoS Biology, 15(3), e2000797.

Van Dessel, P., Hughes, S., \& De Houwer, J. (2018a). How Do Actions Influence Attitudes? An Inferential Account of the Impact of Action Performance on Stimulus Evaluation. Personality and Social Psychology Review.

Van Dessel, P., Hughes, S., \& De Houwer, J. (2018b). Consequence-Based Approach-Avoidance Training: A New and Improved Method for Changing Behavior. Psychological Science, 29, 1899-1910.

Van Dessel, P., Ye, Y., \& De Houwer, J. (2018). Changing Deep-rooted Implicit Evaluation in the Blink of an Eye: Negative Verbal Information Shifts Automatic Liking of Gandhi. Social Psychological and Personality Science. 
Wiers C.E., Gladwin T.E., Ludwig V.U., et al. (2017) Comparing three cognitive biases for alcohol cues in alcohol dependence. Alcohol, 52, 242-248. 